\title{
Percepção dos idosos sobre as ações de educação em saúde desenvolvidas
}

\author{
na atenção básica
}

\author{
Pollyana Barbosa de Lima ${ }^{1}$ \\ Andrea Sugai Mortoza ${ }^{2}$ \\ Edna Regina Silva Pereira ${ }^{3}$
}

\begin{abstract}
RESUMO
O Ministério da Saúde define como porta de entrada a Atenção Básica e como prioridade a realização de ações de educação em saúde para otimizar a saúde da pessoa idosa. Trata-se de uma pesquisa qualitativa exploratória realizada com idosos na Atenção Básica que objetivou verificar o conhecimento dos idosos sobre educação em saúde com ênfase nas facilidades e dificuldades na realização de ações educativas. A técnica de coleta de dados foi o grupo focal. Utilizou-se a Análise de Conteúdo Temática, como proposta por Bardin, para análise de dados. Participaram dos grupos focais 15 idosos. Dos grupos focais emergiram 4 categorias: Educação como comportamento versus educação como ferramenta; $\mathrm{O}$ aprendizado e as trocas de experiências dos idosos nas atividades educativas; Características pessoais que interferem na participação de ações de educação em saúde; descobrindo a educação em saúde como mecanismo para alcançar a qualidade de vida. A maioria dos idosos remetem conceito de educação em saúde ao ato do profissional de saúde ser cortês e educado e pouco como ferramenta para aquisição de conhecimento. Atribuem grande importância ao sentimento de acolhimento e pertencimento aos grupos de educação. Apontam como facilitadores no processo de educação em saúde a proximidade das unidades de saúde com suas residências a disponibilidade e tempo. As dificuldades na realização e adesão às atividades educativas relatadas pelos idosos estão vinculadas às barreiras para locomoção, a falta de incentivo por parte de familiares e cuidadores e a não adaptação às atividades propostas
\end{abstract}

Palavras-chave: Educação em saúde. Atenção básica em saúde. Idosos

\section{Perception of elderly people on health education actions developed in basic}

\section{attention}

\section{ABSTRACT}

The Ministry of Health defines as a gateway to Basic Care and as a priority the implementation of health education actions to optimize the health of the elderly. It is a qualitative exploratory research carried out with elderly people in Primary Care that aimed to verify the knowledge of the elderly about health education with emphasis on the facilities and difficulties in carrying out educational actions.

\footnotetext{
'Pollyana Barbosa de Lima: Mestre em Ensino na Saúde pela Universidade Federal de Goiás (2019). E-mail: pollyana.lima13@gmail.com

${ }^{2}$ Andrea Sugai Mortoza: Doutora em Política Social pela Universidade de Brasília UNB (2011), Professora do curso de Nutrição da Universidade Federal de Goiás UFG. E-mail: andreasugai@gmail.com

${ }^{3}$ Edna Regina Silva Pereira: Doutora em Nefrologia pela Universidade de São Paulo (2000), Pós Doutorado em Educação Médica pela Universidade de Campinas UNICAMP (2013), Professora no curso de Medicina da Universidade Federal de Goiás. E-mail: ersp13@gmail.com
} 


\section{Programa de Pós-Graduação \\ em Ensino na Saúde - MEPES/UFG \\ DOSSIÊ \\ ENSINO NA SAÚDE}

V.17, N.01, 2021. ISSN: 1807-9342

The research instrument used was the focus group. The Thematic Content Analysis, as proposed by Bardin, was used for data analysis. Fifteen elderly people participated in the focus groups. Of the focus groups emerged 4 categories: Education as behavior versus education as a tool; The learning and exchange of experiences of the elderly in educational activities; Personal characteristics of the age that interfere in the participation of health education actions; Discovering health education as a mechanism to achieve quality of life. The elderly attribute the concept of health education to the act of being courteous and educated and not as a tool for acquiring knowledge. They attach great importance to the feeling of welcoming and belonging to the education groups. They point out as facilitators in the process of health education the proximity of the health units with their residences the availability and time and feeling of welcome by the group. The difficulties in carrying out educational activities with the elderly population are linked to the barriers to locomotion and lack of incentive and knowledge of the importance. It was found that most of the interviewees do not have the concept of health education as a process of knowledge construction. The elderly attach great importance to the reception and belonging generated by health education groups.

Keywords: Health education. Primary health care. Elderly

\section{INTRODUÇÃO}

O envelhecimento da população Brasileira se mostra como um dos grandes desafios do século XXI. Dados do IBGE mostram que no ano de 2017 tinha 28 milhões de idosos $(13,5 \%)$ da população. Estima-se que 2031, o percentual de pessoas idosas vai superar pela primeira vez o de crianças e adolescentes de zero a 14 anos. A projeção do IBGE é que, em 2042, o número de idosos represente cerca de um quarto da população nacional (IBGE, 2018).

Pensando não somente no quadro demográfico, o envelhecimento populacional brasileiro imprimiu importantes modificações epidemiológicas e sociais no território nacional, principalmente em virtude do aumento das doenças crônicas não transmissíveis. Tal realidade, quer seja referente ao adoecimento do idoso, quer seja por sua função social desenvolvida, afeta o Sistema Único de Saúde (SUS) que busca, por meio de políticas públicas, responder às demandas desta parcela da população (MEDEIROS; COURA; FERREIRA, 2017).

Com o objetivo de estimular a participação ativa dos idosos na sociedade, a promoção da saúde e bem-estar na velhice e a criação de um ambiente propício ao envelhecimento em 2006 foi aprovada a Política Nacional de Saúde da Pessoa Idosa (PNSPI), formulada pelo Ministério da Saúde, por meio da portaria de $n^{\circ}$ 2.528/2006 (BRASIL, 2006). 


\section{Programa de Pós-Graduação \\ em Ensino na Saúde - MEPES/UFG \\ DOSSIÊ \\ ENSINO NA SAÚdE}

ITINERARIUS

REFLECIONIS

V.17, N.01, 2021. ISSN: 1807-9342

Entre os desafios às políticas públicas voltados para o envelhecimento populacional, presentes na Política Nacional de Saúde da Pessoa Idosa (PNSPI) (2006), encontram-se a prevenção de doenças, a manutenção da saúde, a independência e a autonomia da população idosa. Na tentativa de superação destes desafios a PNSPI definiu que a atenção à saúde da população idosa tem como porta de entrada a Atenção Básica a Saúde (ABS) e o Programa Saúde da Família (PSF) (BRASIL, 2006; Sarreta, 2009).

A Política Nacional de Atenção Básica (PNAB), de 2017, define como atribuições básicas de profissionais da $\mathrm{ABS}$ a realização de ações que promovam a melhoria da qualidade de vida, manutenção da capacidade funcional e o aumento da resolutividade da atenção a pessoa idosa. As ações de educação em saúde realizadas na ABS estão presentes na (PNAB e são realizadas com o objetivo de fortalecer as ações de promoção da saúde e aproximar os profissionais de saúde da comunidade (BRASIL, 2017).

Os profissionais da saúde da ABS utilizam ações de educação em saúde, como grupos do programa de hipertensão arterial sistêmica e diabetes mellitus (HIPERDIA), grupos de caminhada, reuniões para controle de peso, educação em saúde bucal dentre outros, para promover a saúde do idoso e fazer com que o envelhecimento seja saudável e ativo, como preconizado nas políticas públicas de saúde. O foco das ações educativas desenvolvidas na ABS está na diminuição da vulnerabilidade e dos riscos à saúde da população, por meio da participação do idoso de forma ativa expondo suas demandas e necessidades em saúde (MENDONÇA et al., 2017).

Segundo a PNAB os profissionais inseridos na ABS devem facilitar a participação do idoso a grupos de terceira idade e grupos de convivência com ações de promoção e valorização de experiências positivas no conselho de saúde locais, onde os idosos possam ser ouvidos a partir do reconhecimento e incorporação de suas crenças e valores culturais (BRASIL, 2017).

As ações educativas em saúde voltadas para a promoção da saúde do idoso são, na maioria das vezes, vinculadas a programas educativos de sobre atividade física, nutrição e 


\section{Programa de Pós-Graduação \\ em Ensino na Saúde - MEPES/UFG \\ DOSSIÊ \\ ENSINO NA SAÚDE}

ITINERARIUS

REFLECIONIS

V.17, N.01, 2021. ISSN: 1807-9342

doenças crônicas, com palestras e aulas. Alguns exemplos apresentam a inserção de idosos de forma ativa em rodas de conversas; oficinas com orientações e recomendação, recorte e colagem, dramatização, aconselhamento realizado em grupo (ALMEIDA; MOUTINHO; LEITE, 2014; CAMPOS et al., 2012; MENDONÇA et al., 2017; PATROCÍNIO; PEREIRA, 2013).

As atividades grupais são intervenções educativas que podem promover a interação social e auxiliar na melhoria da qualidade de vida (RUMOR et al., 2010; MARTINS et al., 2016). Os idosos que participam de grupos de educação em saúde estreitam vínculos, tanto com os seus pares como com os profissionais de saúde e desenvolvem ações em conjunto. Os grupos representam um dos recursos com maior capacidade de gerar impactos positivos para a saúde do idoso contribuindo para o maior cuidado pessoal e quebra de paradigmas sobre o processo de envelhecimento (AlVES, 2005; CAMPOS et al., 2012).

As estratégias utilizadas na ABS devem ser prioritariamente, fundamentadas em uma relação dialógico-reflexiva entre profissionais de usuários. Devem enfatizar a participação do idoso em todas as etapas do processo educativo empoderando o indivíduo para que este possa decidir sobre o que é melhor para sua saúde (ALMEIDA; MOUTINHO; LEITE, 2014). Observa-se, no entanto, que a maioria das ações educativas desenvolvidas na ABS, ainda são pautadas na prescrição de hábitos utilizando técnicas de coerção e imposição de conhecimentos (SANTOS, 2010).

No Brasil, apesar das ações de educação em saúde estarem presentes na Política Nacional de Atenção Básica (PNAB) e na Política Nacional de Saúde da Pessoa Idosa (PNSPI), como atributos dos profissionais da ABS população idosa, conforme planejamento da equipe e utilizando abordagens adequadas às necessidades deste público, ainda são escassas as informações sobre o conhecimento destes indivíduos sobre aspectos relacionados à educação em saúde, suas aplicações e finalidades. Observe a inserção das siglas no texto!

O baixo conhecimento sobre educação em saúde pode resultar no desinteresse à participação e baixa adesão do idoso às atividades de educação em saúde e comprometer o 


\section{Programa de Pós-Graduação \\ em Ensino na Saúde - MEPES/UFG \\ DOSSIÊ \\ ENSINO NA SAÚDE}

ITINERARIUS

REFLECIONIS

V.17, N.01, 2021. ISSN: 1807-9342

desenvolvimento de políticas públicas e o investimento em estratégias de educação em saúde para a população idosa na ABS.

O objetivo deste estudo foi identificar o conhecimento de idosos atendidos na Atenção Básica sobre educação em saúde e as facilidades e dificuldades na realização das ações de educação saúde na ABS.

\section{PERCURSO METODOLÓGICO}

Com a finalidade de analisar a percepção de um grupo sobre um assunto específico, utilizou-se a abordagem de estudo qualitativa. Para coleta de dados foi utilizada a técnica de grupo focal, com análise dos dados por meio da análise de conteúdo temática de Bardin (2011).

Os critérios de inclusão para o estudo foram: ter mais de 60 anos de idade, ter ficha de cadastro em uma das cinco Unidades Básicas de Saúde de um município de Goiás, ser participante assíduo (maior número de frequência nas atividades educativas por pelo menos seis meses) e pouco participante (menor número de frequência nas atividades educativas por pelo menos seis meses) das ações de educação em saúde realizadas nas unidades pesquisadas. Os critérios de exclusão foram os idosos que abandonaram as atividades educativas sem nenhuma frequência nos últimos seis meses e aqueles que não possuíam capacidade de fala e compreensão para a participação nos grupos focais

Inicialmente 28 ligações foram feitas para convidar 14 idosos que mais participavam dos grupos de educação em saúde (considerados mais assíduos) e 14 ligações para os participantes que menos participavam das atividades (considerados menos assíduos). Dos 14 idosos mais assíduos, nove aceitaram participar dos grupos, já dos 14 idosos menos assíduos, seis idosos aceitaram participar dos grupos focais.

Dois grupos focais foram realizados, no período de novembro de 2017 a janeiro de 2018, com oito participantes no primeiro grupo e sete participantes no segundo grupo focal. 


\section{Programa de Pós-Graduação \\ em Ensino na Saúde - MEPES/UFG \\ DOSSIÊ \\ ENSINO NA SAÚDE}

ITINERARIUS

REFLECIONIS

V.17, N.01, 2021. ISSN: 1807-9342

Os dois grupos focais foram realizados em ambiente amplo e silencioso visando garantir a privacidade e um ambiente acolhedor para os idosos.

Os dois grupos focais foram formados de modo que ambos contivessem participantes considerados assíduos e menos assíduos nas atividades de educação em saúde das unidades. Tal divisão permitiu a formação de grupos focais com características homogenias (por se tratar de idosos de ambos os sexos) e características heterogenias (com diferentes frequências nos grupos).

Os sujeitos participantes da pesquisa foram identificados com as siglas GF1 e GF2 representando o grupo focal do qual fizeram parte. Também foram numerados de forma a identificá-los segundo sua ordem de disponibilização espacial em volta da mesa em sentido horário, na sala onde foram realizados os grupos focais.

A análise de dados foi feita por meio da técnica de análise de conteúdo, descrevendo e interpretando de forma objetiva e sistemática conteúdo advindo dos grupos focais. Dentre as várias modalidades de análise de conteúdo, optou-se pela análise de conteúdo temática fundamentada em Bardin (2011).

As expressões indutoras e as perguntas presentes nos grupos focais foram agrupadas no Quadro 1 segundo a ordem em que foram apresentadas durante os grupos focais, possibilitando a comparação e análise com os termos que emergiram pelos participantes.

Quadro 1 - Quadro com os termos e perguntas utilizadas como roteiro para os grupos focais

\begin{tabular}{|l|l|}
\hline Tema principal & Palavra indutora: educação \\
\cline { 2 - 2 } & Palavra indutora: saúde \\
\cline { 2 - 2 } & $\begin{array}{l}\text { Pergunta: O que vocês acham que significa educação em } \\
\text { saúde? }\end{array}$ \\
\hline
\end{tabular}




\begin{tabular}{|c|l|}
\hline \multirow{2}{*}{$\begin{array}{c}\text { Facilidades e } \\
\text { dificuldades }\end{array}$} & $\begin{array}{l}\text { Pergunta: quais os aspectos que facilitam as ações de } \\
\text { educação em saúde }\end{array}$ \\
\cline { 2 - 2 } & $\begin{array}{l}\text { Pergunta: Quais os aspectos que dificultam as ações de } \\
\text { educação em saúde? }\end{array}$ \\
\cline { 2 - 2 } & Palavra indutora: o que é difícil? \\
\hline Mudanças observadas idosos & Expressão indutora: Qualidade de vida/ estilo de vida \\
\cline { 2 - 2 } & $\begin{array}{l}\text { Pergunta indutora: observam mudanças na qualidade de vida } \\
\text { participando das ações de educação em saúde? }\end{array}$ \\
\hline
\end{tabular}

Fonte: Autores (2018)

As categorias e subcategorias foram definidas com base nos objetivos de estudo e no roteiro utilizado para condução do grupo focal, bem como das falas e conteúdo que emergiram.

O presente estudo foi aprovado pelo Comitê de Ética em Pesquisa da Universidade Federal de Goiás sob parecer de número: 2.259818.

\section{RESULTADOS E DISCUSSÃO}

De um total de 15 idosos participantes da pesquisa, 11 eram do sexo feminino com idade entre 60 e 70 anos e quatro eram do sexo masculino com idade entre 60 e 78 anos. Quanto ao nível de escolaridade dois eram analfabetos e os demais não completaram o ensino fundamental. Moram com o cônjuge oito deles, seis moram com filhos ou outros familiares, e um mora sozinho. Tais dados podem ser observados abaixo no Quadro 2:

Quadro 2 - Perfil sociodemográfico dos idosos que participaram dos grupos focais: Senador Canedo/ GO, 2018 
V.17, N.01, 2021. ISSN: 1807-9342

\begin{tabular}{|l|l|l|l|}
\hline Variável & Categoria & Sujeito & Frequência \\
\hline Sexo & Masculino & 4 & $27 \%$ \\
& Feminino & 11 & $73 \%$ \\
\hline Idade & $60-70$ & 13 & $87 \%$ \\
& $70-80$ & 2 & $13 \%$ \\
\hline escolaridade & Analfabeto & 2 & $13 \%$ \\
& Ensino & 13 & $87 \%$ \\
& Fundamental & & $7 \%$ \\
\hline Com quem reside & Sozinho & 1 & $53 \%$ \\
& Cônjuge & 8 & $33 \%$ \\
& Filho & 5 & $7 \%$ \\
& Outros & 1 & \\
\hline
\end{tabular}

Fonte: Os autores (2018)

A análise do conteúdo resultou em quatro categorias classificadas a partir da identificação no discurso dos idosos participantes expostas no Quadro 3:

Quadro 3 - Síntese da categorização dos dados dos grupos focais com idosos atendidos na ABS, Senador Canedo/GO, 2018:

\begin{tabular}{|c|c|c|}
\hline CATEGORIAS & SUBCATEGORIAS & CÓDIGOS \\
\hline $\begin{array}{lr}\text { Educação } & \text { como } \\
\text { comportamento } & \text { versus } \\
\text { educação como ferramenta }\end{array}$ & $\begin{array}{l}\text { Ato de ser cortês } \\
\text { com o próximo }\end{array}$ & $\begin{array}{l}\text { Tratamento pessoal; } \\
\text { Cortesia; } \\
\text { Bom atendimento e acolhimento }\end{array}$ \\
\hline
\end{tabular}




\begin{tabular}{|c|c|c|}
\hline & $\begin{array}{l}\text { Estratégia utilizada } \\
\text { para aquisição de } \\
\text { novos saberes }\end{array}$ & $\begin{array}{l}\text { Forma de aprender; } \\
\text { Forma de ensinar; } \\
\text { Conhecimento; }\end{array}$ \\
\hline $\begin{array}{l}\mathrm{O} \text { aprendizado e as trocas de } \\
\text { experiências dos idosos nas } \\
\text { atividades educativas }\end{array}$ & & $\begin{array}{l}\text { Cuidado que devemos ter; } \\
\text { Prevenir; } \\
\text { Aprendizado conjunto; } \\
\text { Troca de experiências; }\end{array}$ \\
\hline $\begin{array}{l}\text { Características próprias da idade } \\
\text { que interferem na participação } \\
\text { em ações de educação em saúde }\end{array}$ & $\begin{array}{l}\text { dificultadoras } \\
\text { facilitadoras }\end{array}$ & $\begin{array}{l}\text { Dependência de outros ou } \\
\text { limitação física /doença; } \\
\text { Falta de identificação ou adaptação } \\
\text { das ações propostas; } \\
\text { Distância da Unidade de Saúde: } \\
\text { Disponibilidade de tempo; } \\
\text { Proximidade com a Unidade de } \\
\text { saúde; } \\
\text { Momento de convivência com } \\
\text { outros idosos }\end{array}$ \\
\hline $\begin{array}{l}\text { Descobrindo a educação em } \\
\text { saúde como mecanismo para } \\
\text { alcançar qualidade de vida }\end{array}$ & & $\begin{array}{l}\text { Adoção de novos hábitos de vida; } \\
\text { Sentimento de pertencimento a um } \\
\text { grupo; } \\
\text { Ocupação do tempo }\end{array}$ \\
\hline
\end{tabular}

Fonte: Autores (2018) 


\section{Programa de Pós-Graduação \\ em Ensino na Saúde - MEPES/UFG \\ DOSSIÊ \\ ENSINO NA SAÚDE}

V.17, N.01, 2021. ISSN: 1807-9342

Primeira categoria: Educação como comportamento versus educação como ferramenta para aprendizagem

Alguns idosos apresentaram um conceito inicial de educação em saúde como a forma com que os trabalhadores das unidades de saúde e, até mesmo, os pacientes atendidos nas unidades, comportavam-se frente uns aos outros:

"Eu acho que educação é algo muito importante, as pessoas têm que tratar as outras de forma adequada seja onde for [...] Eu acho que aqui no posto sou tratado com educação, nunca me trataram mal aqui sempre fui bem recebido" (GF1- 6)

Após exemplificação de atividades de educação em saúde com imagens e cartazes contendo ilustrações sobre atividades desenvolvidas nas próprias unidades participantes, os idosos iniciaram um conceito de educação em saúde vinculado à ações e práticas desenvolvidas nas unidades como ferramenta para promoção da saúde e qualidade de vida.

"Acho que educação desta forma é o que a gente aprende aqui, não é? [...] Tem uma placa na entrada aqui falando que no postinho tem educação em saúde, uma vez a enfermeira me disse que era aquelas reuniões do HIPERDIA" (GF1-4)

Percebe-se que os idosos identificam ações das quais participam e que interpretam como ações de educação em saúde. Porém, os exemplos de ações educativas remetidos pelos idosos foram: palestras, folders educativos e orientações durante as consultas. Os exemplos dados e a forma como as ações foram descritas, por meio da transmissão de conhecimento oriunda dos profissionais de saúde para os idosos, caracterizam o modelo de educação em saúde tradicional não dialógico. 


\section{Programa de Pós-Graduação \\ em Ensino na Saúde - MEPES/UFG \\ DOSSIÊ \\ ENSINO NA SAÚDE}

ITINERARIUS

REFLECIONIS

V.17, N.01, 2021. ISSN: 1807-9342

A postura não dialógica da educação em saúde ainda prescritiva dos profissionais de saúde, observada por alguns participante do grupo focal, é semelhante os resultados encontrados no estudo de Ramôa et al., (2017) com mulheres hipertensas. As participantes do estudo, após ingressarem em atividades educativas com ênfase na participação ativa sobre a elaboração e desenvolvimento das atividades e construção de conhecimento compartilhado e dialógico, mostraram-se mais interessadas e aumentaram sua adesão ao tratamento da hipertensão.

Almeida, Moutinho e Leite (2014) observaram em seu estudo, que parte dos usuários não eram habituados ao conceito de educação em saúde mais profundo e a possibilidade de se aprender e ensinar durante as atividades educativas. Após a intervenção proposta pelas autoras, de conscientização sobre as ações educativas, os usuários passaram a ter mais consciência sobre o processo de educação em saúde e sua importância.

Segunda categoria: o aprendizado e as trocas de experiências dos idosos nas atividades educativas.

As ações de educação em saúde são ferramentas utilizadas para trocas de saberes, a fim de favorecer a autonomia, direcionando o cuidado ao idoso para as suas reais necessidades. As abordagens devem basear-se nas demandas dos indivíduos, compreendendo os determinantes biopsicossociais de forma integral, não somente com ênfase na doença apresentada (FERNANDES; BACKERS, 2010).

"Eu venho sempre nos encontros com a enfermeira para o diabetes e também eu venho naquelas reuniões da dentista que ela explica sobre o cuidado que a gente deve ter com os dentes [...] a gente que é mais velho tem que cuidar do que resta da saúde, pra não ter que depender de hospital quando já estiver doente” (GF1-7) 


\section{Programa de Pós-Graduação \\ em Ensino na Saúde - MEPES/UFG \\ DOSSIÊ \\ ENSINO NA SAÚDE}

ITINERARIUS

REFLECIONIS

V.17, N.01, 2021. ISSN: 1807-9342

Nota-se a consciência do idoso da importância do autocuidado e da prevenção de doenças e como os grupos de educação em saúde são importantes para a sensibilização desta população. A educação pode ser utilizada como instrumento para desenvolver nos indivíduos consciência de seus problemas de saúde e de sua responsabilidade em seu cuidado. Estudo de Ferreti et al., (2014), também observaram que os indivíduos idosos que participavam de ações de educação em saúde, demonstravam apropriação de conhecimento sobre o sistema cardiovascular, estabelecendo relações entre a prevenção e cuidados necessários para minimizar os fatores de risco.

Almeida, Moutinho e Leite (2014) também identificaram em seu estudo com idosos hipertensos, relatos que caracterizavam trocas de experiências e aprendizagem construídas durante as atividades de educação em saúde em grupos. As autoras observaram, ainda, que os idosos que participavam de tais atividades partilhavam de experiências semelhantes, sejam com relação à doença ou mesmo ao tratamento e construíam novos conceitos e perspectivas com relação ao envelhecimento.

Há diversas vantagens na realização de atividades educacionais em grupos de idosos como o envolvimento dos integrantes do grupo, a aprendizagem coletiva para o cuidado além da multiplicação de informações. Os idosos, orientam outros idosos e até mesmo familiares que não participam dos grupos educacionais quanto ao conteúdo abordado e aplicabilidade no dia a dia, promovendo o auto cuidado entre semelhantes (MARQUES., et al, 2019).

No fragmento de entrevista abaixo, a participante refere que adquiriu conhecimento sobre alimentação e cuidados com a saúde nos grupos de educação em saúde na diabetes. Destaca-se a presença do aprendizado e troca de saberes com os demais idosos que também participam do grupo.

"Quando tem reunião vem muita gente que eu não tenho contato, aqueles vizinhos mais de longe [...] a gente aprende muita coisa com os outros do grupo. Consegui até algumas receitas na reunião do diabete 


\section{Programa de Pós-Graduação \\ em Ensino na Saúde - MEPES/UFG \\ DOSSIÊ \\ ENSINO NA SAÚDE}

V.17, N.01, 2021. ISSN: 1807-9342

que quem me passou não foi enfermeira foi uma vizinha, e deu certo estou bem melhor do diabete" (GF2-3)

O processo dialético de ensino aprendizagem é enriquecedor durante as práticas de educação em saúde. O profissional, embora seja visto como "professor" no cenário da ABS, adquire conhecimentos pertencentes ao universo dos demais participantes que vão além do saber adquirido no cotidiano da assistência (PINTO; CYRINO, 2015).

A educação em saúde deve ser capaz de utilizar dados advindos de aspectos socioculturais e particulares da população a qual é destinada, considerando as diferenças e semelhanças entre os indivíduos e promovendo a inclusão e participação ativa. Para a promoção da saúde dos idosos as práticas de educação em saúde devem utilizar metodologia reflexiva com ênfase e semelhanças com atividades de vida diárias e demandas pelos idosos, bem como levar em consideração as influências de gênero e cultura (VERAS, 2013).

Terceira categoria: Características próprias da idade que interferem na participação em ações de educação em saúde

Os idosos em seus discursos, por diversas vezes, atribuem aspectos relacionados ao envelhecimento como barreiras para a participação e interesse nas ações de educação em saúde propostas pelas unidades de saúde. Tal aspecto pode ser percebido na seguinte fala da participante que foi incluída na pesquisa por ser uma das usuárias que menos participam das ações de educação em saúde propostas em sua unidade de saúde de origem:

"Sabe como é né. Eu até gostaria de ir acho que seria bom, mas eu não consigo ir sozinha. É longe da minha casa e nestes horários que 


\section{Programa de Pós-Graduação \\ em Ensino na Saúde - MEPES/UFG \\ DOSSIÊ \\ ENSINO NA SAÚDE}

ITINERARIUS

REFLECIONIS

V.17, N.01, 2021. ISSN: 1807-9342

eles fazem as reuniões e chamam a gente as minhas filhas estão fora de casa e não podem me acompanhar. Eu não posso ir sozinha se não acontece alguma coisa e fica é pior" (GF2-2).

As limitações físicas e sociais interferem diretamente na inclusão da pessoa idosa, seja em atividades vinculadas a saúde, seja em atividades socioeducativas. A incapacidade gerada pelo envelhecimento não é um aspecto individual, mas um resultado entre diversos fatores que incluem características especificas, como a presença de doenças crônicas e deficiência de estruturas e função, as questões sociais, as diferenças culturais e a dificuldade de acesso aos serviços de saúde, entre outros fatores (STUCKI; REINHARDT; BICKENBACH, 2015).

"Eu sinto muitas dores minha filha, eu até gostaria de ir no grupo de caminhada, mas eu participei uma vez só e não consegui mais ir [...] Os outros conseguem acompanhar, eu não. Sinto que até atrapalho as vezes..." (GF2-6)

No fragmento de entrevista acima, pode-se perceber o descontentamento da idosa em não poder participar das atividades de educação em saúde por não possuir condições físicas. Nota-se a preocupação da entrevistada que, para não atrapalhar os demais participantes do grupo prefere não ir as atividades.

Em estudo qualitativo com foco na atenção integral a saúde do idoso na ESF, os autores identificaram que os idosos participantes da pesquisa possuem dificuldades no acesso e deslocamento aos serviços de saúde devido a topografia da região e as enfermidades apresentadas por eles. Tais dificuldades impactam diretamente no desenvolvimento de ações de prevenção e promoção da saúde para esta população (DA COSTA; CIOSAK, 2010). 


\section{Programa de Pós-Graduação \\ em Ensino na Saúde - MEPES/UFG \\ DOSSIÊ \\ ENSINO NA SAÚDE}

V.17, N.01, 2021. ISSN: 1807-9342

Mudanças associadas ao processo de envelhecimento bem como a presença de certas patologias afetam as esferas físicas, cognitiva e psicossocial. Tais alterações podem modificar funções sensoriais, memória, pensamento logico, nível de energia e resistência física afetando a capacidade do idoso de autogerir sua doença (GIROUX; ELLEFSEN; MAILHOTBISSON, 2020)

Um ambiente físico com características ambientais adequadas, segurança e proximidade com os serviços de saúde foram associados à melhora da qualidade de vida de idosos, influenciando diretamente em sua saúde e bem-estar. Por outro lado, dificuldades para sair de casa de forma independente, barreiras físicas e distância dos centros de saúde são apontados como aumento de incapacidade de idosos, resultando em isolamento social (NASCIMENTO et al., 2018; PORTEGIJS et al., 2017).

Uma facilidade citada pelos idosos para a participação de atividades de educação em saúde foi a disponibilidade de tempo para a participação de atividades educativas como pode-se observar nas falas abaixo:

"Eu acho que para mim é mais fácil participar porque eu não trabalho e lá em casa sobra tempo quando eu não tenho que cuidar dos meus netos. Tem gente do grupo que trabalha fora ainda, ai eles faltam as reuniões porque são no meio da tarde sabe?" (GF2-1)

"Aqui é bem perto da minha casa e eu acho muito bom vir para conversar com os doutores e ver as minhas colegas. Para mim não é esforço nenhum, eu venho sozinha e não gasto nem 10 minutos [...] pode ver ai no meu cartão, eu nunca faltei” (GF2-3) 


\section{Programa de Pós-Graduação \\ em Ensino na Saúde - MEPES/UFG \\ DOSSIÊ \\ ENSINO NA SAÚDE}

ITINERARIUS

REFLECIONIS

V.17, N.01, 2021. ISSN: 1807-9342

Em estudo realizado em 2014 com idosos participantes de atividade física identificou melhor percepção de qualidade de vida dos idosos a disponibilidade de tempo para a realização de atividades educativas e físicas. Para os entrevistados, o processo de envelhecimento em si, não afetou de maneira negativa a percepção de qualidade de vida (AMARAL, 2012). Pereira e Okuma (2009), também observaram em seu estudo a ocupação do tempo livre como um dos motivos de adesão de idosos a um programa de educação física para indivíduos da terceira idade.

Outra característica apontada como facilitadora para a participação dos grupos de educação em saúde pelos idosos entrevistados foi o sentimento de inclusão e pertencimento ao grupo:

"Eu gosto muito de vir para os grupos [..] Vejo todos os meus colegas, os vizinhos[...] A gente se sente acolhido nos grupos [...] participo de um que estamos juntos já tem quase um ano, nos vemos todas as semanas e podemos conversar [...] A enfermeira [...] está com a gente neste um ano. Ela já sabe muito sobre a gente e consegue ajudar em muitas coisas. (GF2-5).

Pode-se perceber a importância do sentimento de inclusão em grupo, do acolhimento pelos profissionais de saúde e pelos participantes dos grupos que geram atitudes positivas quanto à participação e aproveitamento nas atividades de educação em saúde propostas. Perceberam nas falas de usuários da ABS a importância da formação do vínculo, no contexto dos grupos de educação em saúde, para adesão e permanência dos participantes dos grupos e otimização dos resultados (FRIEDRICH et al., 2018).

Observa-se que geralmente programas de educação são construídos de tal forma a atender as necessidades de crianças, adolescentes e jovens adultos em sua grande maioria. 


\section{Programa de Pós-Graduação \\ em Ensino na Saúde - MEPES/UFG \\ DOSSIÊ \\ ENSINO NA SAÚDE}

V.17, N.01, 2021. ISSN: 1807-9342

Alguns países como a China, no entanto, dispõem de recursos e serviços para os idosos a fim de garantir o processo de educação em saúde para esta população sendo as atividades realizadas em grupo peça chave para tal processo ( GIROUX; ELEFSSEN; MAILHOT-BISSON, 2020).

Valer et al., (2015), identificaram que, para os idosos, a criação de rede de amizades a partir do convívio e das oportunidades proporcionadas são fatos importantes para a sua participação nas ações propostas. As atividades de educação em saúde, realizadas em grupos, foram destacadas como uma oportunidade de aprendizado e troca com outras pessoas idosas e profissionais de saúde.

Quarta categoria: A educação em saúde como promotora da qualidade de vida.

Os idosos, após envolvimento no grupo focal e criação e recriação de novos significados referentes à educação em saúde, identificaram como principais benefícios de sua participação, a adoção de hábitos mais saudáveis e a socialização com indivíduos com realidades próximas, impactando diretamente na qualidade de vida.

"O melhor de participar dos grupos aqui é que eu conheço muita gente que tem o mesmo problema que eu [...] quando eles falam algo novo pra gente a gente começa a fazer, todo mundo muda junto, e muda para melhor[...] No nosso grupo do diabete muita gente emagreceu desde o começo do ano [...] estou empolgada para ver onde a gente vai conseguir chegar". (GF2-5)

“ Ah, eu me sinto bem melhor desde que eu comecei a participar do grupo da doutora [...] ela me disse que era para eu parar meu café de noite e falou para eu não tomar tudo junto dos remédios da pressão [...] eu não conseguia dormir do jeito que eu durmo hoje (GF1-7) 


\section{Programa de Pós-Graduação \\ em Ensino na Saúde - MEPES/UFG \\ DOSSIÊ \\ ENSINO NA SAÚDE}

ITINERARIUS

REFLECIONIS

V.17, N.01, 2021. ISSN: 1807-9342

Estudo de abordagem qualitativa e participativa com idosos de um grupo de educação em saúde sobre doenças cardiovasculares, observou apropriação de novos conhecimentos acerca do tema com modificações permanentes em hábitos de vida diário e associação entre aspectos preventivos impactando na qualidade de vida dos idosos (FERRETTI et al., 2014)

Similarmente ao conteúdo encontrado nas falas dos idosos do presente estudo, estudos sobre educação em saúde mostram que as atividades de educação em saúde realizadas em grupos podem, ao promover o autocuidado, a valorização da vida, e a busca ativa da saúde, melhorar a qualidade de vida dos idosos (ALMEIDA; MOUTINHO; LEITE, 2016; MELO et al., 2014; MALLMANN et al., 2015; MENDONÇA et al., 2017).

\section{CONSIDERAÇÕES FINAIS}

Poucos são os estudos que apresentam o a visão do idoso usuário do SUS sobre as ações de educação em saúde propostas na ABS.

A maioria dos idosos não possuem o conceito formal de educação em saúde como processo de construção e reconstrução de conhecimento, mas entendem as ações de educação em saúde como alcance de qualidade de vida. Os idosos atribuem grande importância ao acolhimento e pertencimento gerados por grupos de educação em saúde.

As facilidades para a realização de atividades de educação em saúde na atenção básica aos olhos dos idosos, estão relacionadas a proximidade da unidade de saúde com a residência destes bem como os sentimentos positivos gerados durante a realização dos grupos de educação. A interação com indivíduos de mesma faixa etária e que carregam consigo demandas e necessidades semelhantes em saúde, são aspectos positivos que motivam os idosos principalmente a se manter nos grupos de educação em saúde. 


\section{Programa de Pós-Graduação \\ em Ensino na Saúde - MEPES/UFG \\ DOSSIÊ \\ ENSINO NA SAÚdE}

ITINERARIUS

REFLECIONIS

V.17, N.01, 2021. ISSN: 1807-9342

As dificuldades na participação em grupos de educação em saúde estão relacionadas, principalmente as barreiras de locomoção e características próprias do processo de envelhecimento, que fazem com que os idosos dependam de familiares ou conhecidos para que se desloquem até as unidades de saúde e participem das atividades de educação em saúde.

Os resultados deste estudo podem contribuir para elaboração de estratégias dirigidas à população idosa, a serem observadas por profissionais de saúde, gestão e usuários, reduzindo custos relacionados ao tratamento de doenças e agravos ao idoso e suas complicações, melhorando a qualidade de vida para este grupo.

\section{REFERÊNCIAS}

AMARAL, F.L.J.S. et al. Fatores associados com a dificuldade no acesso de idosos com deficiência aos serviços de saúde. Ciência \& Saúde Coletiva, v. 17, p. 2991-3001, 2012.

ALMEIDA, E. R.; MOUTINHO, C. B.; LEITE, M. T. DE S. A prática da educação em saúde na percepção dos usuários hipertensos e diabéticos. Saúde em Debate, v. 38, n. 101, p. 328-337, 2014.

ALMEIDA, E. R.; MOUTINHO, C. B.; LEITE, M. T. DE S. Prática pedagógica de enfermeiros de Saúde da Família no desenvolvimento da Educação em Saúde. InterfaceComunicação, Saúde, Educação, v. 20, n. 57, p. 389-401, 2016.

ALVES, V. S. Um modelo de educação em saúde para o Programa Saúde da Família: pela integralidade da atenção e reorientação do modelo assistencial. Interface - Comunicação, Saúde, Educação, v. 9, n. 16, p. 39-52, 2005.

BARDIN, L. Análise de conteúdo. Tradução de Luis Antero Reto, Augusto Pinheiro. São Paulo: Edições 70, 2011.

BRASIL. Instituto Brasileiro de Geografia e Estatistica. Censo Demográfico, IBGE, 2017. Disponível em < www. Ibge.gov.br>acesso em 12 de março de 2018. 


\section{Programa de Pós-Graduação \\ em Ensino na Saúde - MEPES/UFG \\ DOSSIÊ \\ ENSINO NA SAÚDE}

V.17, N.01, 2021. ISSN: 1807-9342

. Ministério da Saúde. Secretaria de Atenção à Saúde. Departamento de Atenção Básica. Política Nacional de Atenção Básica / Ministério da Saúde. Secretaria de Atenção à Saúde. Departamento de Atenção Básica. - Brasília: Ministério da Saúde, pg:110, 2017.

. Ministério da Saúde. Secretaria de Atenção à Saúde. Departamento de Atenção Básica. Núcleo de Apoio à Saúde da Família / Ministério da Saúde, Secretaria de Atenção à Saúde, Departamento de Atenção Básica. - Brasília: Ministério da Saúde n.39, pg 106, 2014.

. RESOLUÇÃO No 466, DE 12 DE DEZEMBRO DE 2012 (DOU 13/06/2013).

Trata de pesquisas em seres humanos e atualiza a resolução 196/96. Brasília, 2012.

Ministério da Saúde. Diretrizes operacionais dos pactos pela vida, em defesa do

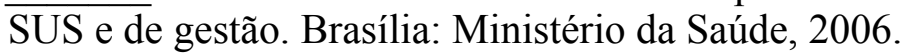

CAMPOS, C. N. A. et al. Reinventing nursing practice in health education: theater with elderly. Anna Nery School Journal of Nursing / Escola Anna Nery Revista de Enfermagem, v. 16, n. 3, p. 588-596 9p, 2012.

DA COSTA, M. F. B. N. A.; CIOSAK, S. I. Atenção integral na saúde do idoso no Programa Saúde da Família : visão dos profissionais de saúde. Revista Escola de Enfermagem USP, v. 44, p. 437-444, 2010.

FERNANDES, M. C. P.; BACKERS, V. M. S. Educação em saúde: perspectivas de uma equipe da Estratégia Saúde da Família sob a óptica de Paulo Freire. Revista Brasileira de Enfermagem, v. 63, n. 4, p. 567-573, 2010.

FERRETTI, F. et al. Impacto de programa de educação em saúde no conhecimento de idosos sobre doenças cardiovasculares. Revista de Saúde Pública, v. 16, n. 6, p. 807-820, 2015.

FRIEDRICH, T. L. et al. Motivações para práticas coletivas na atenção básica: Percepção de usuários e profissionais. Interface - Comunicação, Saúde, Educação, v. 22, n. 65, p. 373-385, 2018.

GIROUX, X.; ELLEFSEN, E.; MAILHOT-BISSON, DIDIER. L'enseignement adapté à la personne âgée : une analyse évolutionniste de concept. Matieres a Reflexion, n. 140, p. $17-28,2020$.

MALLMANN, D. G. et al. Educação em saúde como principal alternativa para promover a saúde do idoso. Ciência \& Saúde Coletiva, v. 20, n. 6, p. 1763-1772, 2015. 


\section{Programa de Pós-Graduação \\ em Ensino na Saúde - MEPES/UFG \\ DOSSIÊ \\ ENSINO NA SAÚDE}

V.17, N.01, 2021. ISSN: 1807-9342

MARQUES, M.B. et al. Intervençao educativa para promoção do autocuidado de idosos com Diabetes Mellitus. Jornal da Escola de Enfermagem da USP, n.53, 2019.

MARTINS, K. L. A. et al. Percepção De Enfermeiros sobre a pratica de educação em saúde na estratégia de saúde da familia. Revista Saúde. Com, v. 12, n. 1, p. 514-520, 2016.

MEDEIROS, K. K. A. S.; COURA, A. S.; FERREIRA, R. T. O aumento do contingente ppulacional e a atenção primária à saude: uma revisão de literatura. Arquivos de Ciências em Saúde UNIPAR, v. 21, p. 201-207, 2017.

MENDONÇA, F. T. N. et al. Educação em saúde com idosos : pesquisa-ação com profi ssionais da atenção primária. Revista Brasileira de Enfermagem, v. 70, n. 4, p. 825-832, 2017.

NASCIMENTO, C. F. DO et al.Fatores individuais e de comunidade associados a mobilidade funcional e queda em idosos residentes em São Paulo. Jornal de Saúde e Envelhecimento, v. 30, n. 1, p. 118-139, 2018.

PATROCINIO, W. P.; PEREIRA, B. DA P. DA C. Efeitos da educação em saúde sobre atitudes de idosos e sua contribuição para a educação gerontológica. Trabalho, Educação e Saúde, v. 11, n. 2, p. 375-394, 2013.

PEREIRA, J. R. P.; OKAMA, S.S. O perfil dos ingressantes de um programa de educação física para idosos e os motivos da adesão inicial. Revista Brasileira de Educação Física e Esporte, v. 23, n. 4, p. 319-334, 2009.

PINTO, T. R.; CYRINO, E. G. Com a palavra, o trabalhador da Atenção primária à Saúde: Potencialidades e desafios nas práticas educacionais. Interface- Comunicação, Saúde, Educação, v. 19, p. 765-777, 2015.

PORTEGIJS, E. et al. Perceived and objective entrance-related environmental barriers and daily out-of-home mobility in community-dwelling older people. Archives of Gerontology and Geriatrics, v. 69, p. 69-76, 2017.

RAMÔA CASTRO, A. et al. Impacto de intervenções educativas na prevenção primária de doença cardiovascular: uma revisão sistemática com foco na atividade física. Jornal Europeu de Praticas Gerais, v. 23, n. 1, p. 59-68, 2017.

RUMOR , P.C.F; BERNS, I.J; HEIDEMANN, I.T.S; MATTOS, L.H.L; WOSNY, A.N. A promoção da saúde nas práticas educativas da saúde da familia. Cogitare Enfermagem, 


\section{Programa de Pós-Graduação \\ em Ensino na Saúde - MEPES/UFG \\ DOSSIÊ \\ ENSINO NA SAÚDE}

V.17, N.01, 2021. ISSN: 1807-9342

v.15, n.4, p: 674-680, 2010.

STUCKI, G.; REINHARDT, J. D.; BICKENBACH, J. Fundamentos Teóricos para a Mensuração de Fatores Ambientais e seu Impacto na Participação de Pessoas com

Deficiência. Arquivos de Medicina Física e Reabilitação, v. 96, n. 9, p. 1739-1740, 2015.

VALER, D. B. et al. O significado de envelhecimento saudável para pessoas idosas vinculadas a grupos educativos. Revista Brasileira de Geriatria e Gerontologia, v. 18, n. 4, p. 809-819, 2015.

VERAS, R. P. Repensando O Sentido Da Prevenção. Revista de Saúde Coletiva, v. 23, p. 1189-1213, 2013. 